\title{
Practitioners and Clinical Trials
}

\author{
True to Your Patients - or Science?
}

$T$ he advancement of human science, particularly medical therapeutics, depends upon clinical trials. However, clinical research in the United States is being scrutinized for a variety of reasons, including perceived shortcomings in the protection of research subjects. ${ }^{1}$ Practicing physicians are an important source for referral of subjects to clinical trials, and yet few studies have actually analyzed how or why they make decisions to encourage their patients to enroll. If we are to have future success in recruiting patients for well-designed and safe clinical trials, we need to understand more about the context in which practicing physicians make decisions about possible referrals.

In this issue, the study by Halpern et al. takes an important step in trying to elucidate those reasons and motivations. ${ }^{2}$ The authors crafted a well-designed study, with a large sample of primary care practitioners who were almost entirely involved in direct patient care, and a detailed mailed survey to clarify what factors practitioners might use in making decisions related to the perceived differences between placebo-controlled trials (PCTs) and active-controlled trials (ACTs).

One of their strongest conclusions is that primary care physicians would prefer to refer their patients to ACTs rather than PCTs because of the perceived benefits to both the patients and themselves. They also found that clinicians are more likely to prescribe new drugs that had been compared in ACTs.

As with all good studies, new questions are raised with the questions that are answered. For example, why are clinicians not only uninterested in placebo trials but also suspicious of the benefits and even morality of these trials? One reason for clinicians' concerns regarding PCTs is that they might not understand what the term and practice really means, or may not realize how many currently used therapeutic agents in our daily armamentarium could not pass the scrutiny of a carefully designed PCT. This confusion is not confined to practitioners, since the placebo effect has again made a comeback after having been reportedly discredited by a Danish study that suggested that placebos were no more effective than doing nothing for a variety of medical illnesses. ${ }^{3}$ Older practitioners might even recall from past decades the use of placebos in everyday practice as an overt act of deception.

Of comfort to patients and the public is the observation that the physicians seemed to focus upon the best interests of those they cared for when making decisions concerning possible referral. Physicians, at least in this study, seemed to truly believe that the treatment of hypertension is accepted medical practice and that not providing it might constitute poor care. They might even have been concerned that they could be held liable if a 738 patient had a known hypertensive complication such as stroke or myocardial infarction during the 3 months that therapy might not have been provided in the hypothetical case example. But are clinicians' concerns about stopping therapy, at least for mild to moderate hypertension, ill founded? A recent meta-analysis of 25 randomized trials examining the efficacy of antihypertensive therapy found that short-term exposure to placebo in these trials did not seem to be associated with an increased risk of serious adverse events. ${ }^{4}$

The primary professional responsibility of the practicing physician is to always place the best interests of the patient first, before the advancement of medical science. As patients also become more sophisticated recipients of medical care, their willingness to forego "possibly beneficial" care could be diminishing. How many men, for example, with hypercholesterolemia would be willing to forgo their statin therapy for several months when they have been saturated with media pronouncements, often drug company initiated, that these drugs can "prevent heart attacks"? And how many informed women would be willing to lengthen the time interval between their routine mammograms as part of a clinical trial, even though the most appropriate interval has not been yet determined? In addition, Institutional Review Boards are increasingly facing similar dilemmas when trying to balance the potential worth of a new or improved medication or invasive intervention against the perception that an existing form of therapy is already "proven."

The additional observation from the study that physicians prefer to consider new drugs in comparison to existing ones should not come as a surprise. Clinicians are inundated with many new versions of drugs and are hard pressed to ascertain significant differences between them. A recent study by the National Institute for Health Care Management Foundation found that of the 1,035 drugs approved by the FDA from 1989 to 2000, only 361, or $35 \%$ contained new active ingredients. The rest contained active ingredients that were already available in other medicines on the market. And the Institute reported that only $15 \%$ could be classified as highly innovative and contained ingredients that offered significant improvements over existing drugs. Although pharmaceutical companies have challenged this report, the reality is that many drugs are quite similar in action and efficacy and the pragmatic practitioner simply wants to know if a new one is better than the one already being used.

In summary, a full discussion of the benefits and limitations of PCTs versus ACTs is well beyond the scope of this editorial, but suffice it to say that PCTs do offer unique advantages in efficiency and in determining 
efficacy for certain drugs in certain situations. But if patient and subject safety is to be maximized and practitioners are to be encouraged to refer their patients to clinical trials, safeguards need to be assured. Clinical studies must be designed to be as low risk as possible and monitoring must be adequate. Results, particularly adverse ones, need to be tracked by a data and safety monitoring process, and withdrawal from a study, if needed, must be expeditious. For practitioners, medical trainees, and academic physicians, a renewed commitment to education about what clinical research truly is and is not is imperative. Not only the science but also the ethics of clinical investigation need to be emphasized so that both researchers and practitioners who might refer patients understand how to balance patient well-being with the goals of research. Ultimately, not only researchers, practitioners, and potential patient-subjects, but funding agencies and the public at large must be reassured that their trust in our entire clinical research enterprise is well placed, secure, and not misguided. Eugene V. BoISAubin, MD, Department of Medicine and General Clinical Research Center, University of Texas School of Medicine at Houston, Houston, Tex.

\section{REFERENCES}

1. Steinbrook R. Improving protection for research subjects. N Engl J Med. 2002;346:1425-30.

2. Halpern SD, Ubel PA, Berlin JA, Townsend RR, Asch DA. Physicians' preferences for active-controlled versus placebocontrolled trials of new antihypertensive drugs. J Gen Intern Med. 2002;17:689-95.

3. Hrobjartsson A, Gotzsche PC. Is the placebo powerless? An analysis of clinical trials comparing treatment with no treatment. N Engl J Med. 2001;344:1594-602.

4. Al-Khatib SM, Califf RM, Hasselblad V, Alexander JH, McCrory DC, Sugarman J. Placebo-controls in short term clinical trials of hypertension. Science. 2001;292:2013-5. 